\title{
VECTOR-VALUED INEQUALITIES WITH WEIGHTS
}

\author{
LUZ M. FERnÁNDEZ-CABRERA AND JOSÉ L. TORREA ${ }^{(*)}$
}

Abstract

This paper deals with the following problem:

Let $T$ be a given operator. Find conditions on $v(x)$ (resp. $u(x)$ ) such that

$$
\int|T f(x)|^{p} u(x) d x \leq C \int|f(x)|^{p} v(x) d x
$$

is satisfied for some $u(x)$ (resp. $v(x)$ ).

Using vector-valued inequalities the problem is solved for: Carleson's maximal operator of Fourier partial sums, LittlewoodPaley square functions, Hitbert transform of functions valued in U.M.D. Banach spaces and operators in the upper-half plane.

\section{Introduction}

This paper deals with the following

Problem A. Let $T$ be a given operator. Find conditions on $v(x)$ (resp. $u(x)$ ) such that

$$
\int|T f(x)|^{p_{u}} u(x) d x \leq C \int|f(x)|^{p} v(x) d x
$$

is satisfied for some $v(x)$ (resp. $v(x)$ ).

This problem was studied for different operators in $[\mathbf{C}, J],[\mathbf{G}, \mathbf{G}],[\mathbf{H}$, M, S]. The operators treated were singular integrals, fractional integrals, Hardy-Littlewood maximal operator and fractional maximal operator. In all the cases the method used was constructive.

(*) Partially sypported by DGICYT. PB90-187. 
On the other hand it was hnown that a "good" weighted norm inequality of type (0.1), for an operator $T$, gives a vector-valued inequality of type

$$
\|\left(\sum_{j}\left[\left.T f_{j}\right|^{p}\right)^{1 / p}\left\|_{s} \leq C\right\|\left(\sum_{j}\left|f_{j}\right|^{p}\right)^{1 / p} \|_{s}\right.
$$

For instance, the vector valued inequalities for the Hardy-Littlewood maximal operator, $M$, were obtained in $[\mathbf{F}, \mathbf{S}]$ from the estimate

$$
\int|M f|^{p} u \leq C \int|f|^{p} M u, \quad 1<p<\infty .
$$

In 1981, José Luis Rubio de Francia showed that weighted norm inequalities and vector valued inequalities were equivalent in some sense, see $[\mathbf{R}$ de $\mathbf{F}, \mathbf{1}]$. Using that equivalence, he developed a non constructive method in order to solve the Problem A for some operators.

The aim of this paper is to show that a slight generalization of the method of Rubio de Francia allows us to solve the Problem A for a huge family of operators. The generalization is two fold:

First we shall consider vector-valued versions of inequality $(0.1)$ and we shall prove the relation with the corresponding inequality $(0.2)$, see Theorem (1.1). We use this vector-valued version in order to:

(a) solve partially Problem A for Carleson's maximal operator of Fourier partial sums, see Theorem (2.9).

(b) find conditions on $v(x)$ (resp. $u(x)$ ) such that

$$
\int_{\mathbf{R}}\|H f(x)\|_{E}^{p} u(x) d x \leq C \int_{\mathbf{R}}\|f(x)\|_{E}^{p} v(x) d x
$$

is satisfied for some $u(x)$ (resp. $v(x)$ ), where $H$ is the Hilbert transform and $E$ is a U.M.D. Banach space, see Theorem (2.24).

In the case that $E$ is a U.M.D. Banach lattice we solve the same problem for the operator

$$
\tilde{M} f(x)=\sup _{x \in Q} \frac{1}{|Q|} \int_{Q}|f(y)| d y, \quad x \in \mathbf{R}^{n}
$$

where |.| is the absolute value in $E$ and the supremum is taken in the lattice order, see Theorem (2.26)

(c) solve Problem A for Littlewood-Paley square functions, see Theorem (2.11). 
The second generalization is to consider abstract measure spaces, instead of $\mathbf{R}^{n}$. With these ideas we are able to solve Problem A for operators which map functions in $\mathbf{R}^{n} \times[0, \infty)$ into functions in $\mathbf{R}^{n} \times(0, \infty)$, see Theorems (2.16) and (2.17). The vector-valued inequalities obtained in this case, see Theorem (3.16), can be of independent interest when working with operators acting on functions defined on the upper half plane. These operators include as particular cases Poisson integrals, balayages, see (2.19), and some well known maximal operators, see (2.23).

Our method gives in all the cases some extra information about the size of the weight that is found.

It is an honour for us to use ideas of our friend and advisor José Luis.

Throughout this paper we shall work on general measure spaces $(Y, d \nu),(X, d \mu)$, where $d \nu$ and $d \mu$ are positive measures. Given a Banach space $E$, we shall denote by $L_{E}^{p}(Y, d \nu), L_{E}^{p}(Y)$ or $L_{E}^{p}(d \nu)$ the Bochner space of $E$-valued strongly measurable functions such that

$$
\int_{Y}\|f(x)\|_{E}^{p} d \nu(x)<+\infty .
$$

Given a positive measurable function $w(x)$, on $(Y, d \nu)$, we shall denote by

$$
L_{E}^{p}(w(x) d \nu(x))
$$

or $L_{E}^{p}(w)$ the space of $E$-valued strongly measurable functions such that

$$
\int_{Y}\|f(x)\|_{E}^{p} w(x) d \nu(x)
$$

is finite.

Given a Banach space $E$, we shall denote by $\ell_{E}^{p}$, or $\ell^{p}(E)$ the Banach space

$$
\left\{\left\{\alpha_{n}\right\} \subset E: \sum_{n=1}^{\infty}\left\|\alpha_{n}\right\|_{E}^{p}<+\infty\right\} .
$$

If $E$ is a Banach lattice we shall denote by $E\left(\ell^{\infty}\right)$ the lattice

$$
\left\{\left\{x_{n}\right\}: \sup \left|x_{n}\right| \in E\right\}
$$

with the norm $\left\|\left\{x_{n}\right\}\right\|_{E(\ell \infty)}=\left\|\sup _{n} \mid x_{n}\right\| \|_{E}$.

The organization of the paper is as follows: in Section 1 we state and prove the abstract results that generalize the previous work of Rubio de Francia, in Section 2 we give (without proof) the applications that solve Problem A for several operators and Section 3 is devoted to the proofs. 


\section{Abstract results}

We begin this section with a lemma, that it is known for the scalar case, see [GC, R de F, VI.4.2]. The proof in the vector-valued case is essencially the same, but we include it here for the sake of completeness.

(1.0) Lemma. Let $(Y, d \nu)$ be a measure space, let $F$ and $G$ be Banach spaces. Assume thot $0<s<p<\infty$ and $T$ is a sublinear operator which satisfies

$$
\left\|\left(\sum_{j}\left\|T f_{j}\right\|_{F}^{p}\right)^{1 / p}\right\|_{L^{s}(Y, d v)} \leq C\left(\sum_{j}\left\|f_{j}\right\|_{G}^{p}\right)^{1 / p}
$$

Then, if $\sigma=\left(\frac{p}{s}\right)^{\prime}$, there exists a nonnegative function $w(x)$ with $\|w\|_{L^{\sigma-1}(Y, d \nu)} \leq 1$ and such that

$$
\int_{Y}\left\|T f_{j}(x)\right\|_{F}^{p} w(x) d \nu(x) \leq C\left\|f_{j}\right\|_{C^{*}}^{p}
$$

The proof of this lemma is based in the following result, see [GC, R de $\mathbf{F}]$.

Mini-max Theorem. Let $A, B$ be convex sets in some vector spaces and assume that $B$ is compact for a certain topology. Let $\phi$ be a function, $\phi: A \times B \rightarrow \mathbf{R} \cup\{+\infty\}$, which is concave on $A$ and conex and lower semicontinuous on $B$. Then

$$
\min _{b \in B} \sup _{a \in A} \phi(a, b)=\sup _{a \in A} \min _{b \in B} \phi(a, b) .
$$

Proof of the lemma (1.0): Let $A$ and $B$ given by

$$
\begin{aligned}
& A=\left\{\sum_{j}\left\|T f_{j}\right\|_{F}^{p}: f_{j} \in G, \sum_{j}\left\|f_{j}\right\|_{G}^{p} \leq 1\right\} \\
& B=\left\{b \in L^{\sigma}(Y): b(x) \geq 0,\|b\|_{\sigma} \leq 1\right\}
\end{aligned}
$$

and we define on $A \times B$ the function $\phi$ as

$$
\phi(a, b)=\int \sum_{j}\left\|T f_{j}(x)\right\|_{F}^{p} b(x)^{-\sigma^{\prime}} d \nu(x),
$$

$B$ is convex and weakly compact, $A$ is convex; $\phi$ is convex and lower semicontinuous in $B$, see VI.4.3 in [GC, $\mathbf{R}$ de $\mathbf{F}$ ], and $\phi$ is linear on $A$, therefore by the Minimax Theorem we have,

$$
\begin{aligned}
\min _{b \in B} \sup _{a \in A} \phi(a, b) & =\sup _{a \in A} \min _{b \in B} \phi(a, b) \leq \\
& \leq \sup _{a \in A}\left\|\left(\sum_{j}\left\|T f_{j}\right\|_{F}^{p}\right)^{1 / p}\right\|_{L^{s}(Y, d \nu)}^{p} \leq C^{p} .
\end{aligned}
$$


Therefore, there exist $b_{0} \in B$ such that, for every $a \in A$, we have

$$
\int \sum_{j}\left\|T f_{j}(x)\right\|_{F}^{p} b_{0}(x)^{-\sigma^{\prime}} d \nu(x) \leq C^{p},
$$

the proof of the lemma finishes by choosing $w(x)=b_{0}(x)^{-\sigma^{\prime}}$.

(1.1) Theorem. Let $(Y, d \nu)$ be a measure space, $F$ and $G$ be $B a-$ nach spaces, and $\left\{A_{k}\right\}_{k=0}^{+\infty}$ be a sequence of disjoint sets in $Y$ such that $\bigcup_{k=0}^{+\infty} A_{k}=Y$. Assume that $0<s<p<\infty$ and $T$ is a sublinear operator which satisfies

$$
\left\|\left(\sum_{j}\left\|T f_{j}\right\|_{F}^{p}\right)^{1 / p}\right\|_{L^{s}\left(A_{k}, d \nu\right)} \leq C_{k}\left(\sum_{j}\left\|f_{j}\right\|_{G}^{p}\right)^{1 / p}, \quad k \in 0,1, \ldots
$$

where for each $k, C_{k}$ is a constant depending on $G, F, p$ and $s$.

Then there exists a positive function $u(x)$ on $Y$ such that

$$
\left(\int_{Y}\|T f(x)\|_{F}^{p} u(x) d \nu(x)\right)^{1 / p} \leq C\|f\|_{G}
$$

holds, where $C$ is a constant depending on $G, F, p$ and $s$.

Moreover, given a double sequence $\left\{a_{k}\right\}_{k=0}^{+\infty}$ such that $\sum_{k=0}^{+\infty} a_{k}^{p}<+\infty$ and $\sigma=\left(\begin{array}{l}2 \\ s\end{array}\right)^{\prime}, u$ can be found such that

$$
\left\|u^{-1} \mathcal{X}_{A_{k}}\right\|_{L^{\sigma-1}\left(A_{k}, d \nu\right)} \leq\left(a_{k}^{-1} C_{k}\right)^{p} .
$$

Proof: Given $k$, we define the operator $T_{k}$ by

$$
T_{k} f(x)=T f(x) \mathcal{X}_{A_{k}}(x), \quad x \in A_{k} .
$$

$T_{k}$ satisfies

$$
\left\|\left(\sum_{j}\left\|T_{k} f_{j}\right\|_{F}^{p}\right)^{1 / p}\right\|_{L^{s}\left(A_{k}, d \nu\right)} \leq C_{k}\left(\sum_{j}\left\|f_{j}\right\|_{G}^{p}\right)^{1 / p}
$$

then by lemma (1.0), there exists a nonnegative function $w_{k}$ defined on $A_{k}$ such that $\left\|w^{-1}\right\|_{L^{\sigma-1}\left(A_{k}, d \nu\right)} \leq 1$ and

$$
\int_{A_{k}}\left\|T_{k} f(x)\right\|_{F}^{p} w_{k}(x) d \nu(x) \leq C_{k}^{p}\|f\|_{G}^{p}
$$


We define $u(x)$ by $u(x)=\sum_{0}^{+\infty} a_{k}^{p} C_{k}^{-p} w_{k}(x) \mathcal{X}_{A_{k}}(x), \quad x \in Y$; then

$$
\begin{aligned}
& \int_{Y}\|T f(x)\|_{F}^{p} u(x) d \nu(x)= \\
& =\sum_{0}^{+\infty} a_{k}^{p} C_{k}^{-p} \int_{A_{k}}\left\|T_{k} f(x)\right\|_{F}^{p} w(x) d \nu(x) \leq \sum_{0}^{+\infty} a_{k}^{p}\|f\|_{G}^{p}= \\
& =C\|f\|_{G}^{p},
\end{aligned}
$$

and $\left\|u^{-1} \mathcal{X}_{A_{k}}\right\|_{L^{\sigma-1}\left(A_{k}, d \nu\right)}=\left(a_{k}^{-1} C_{k}\right)^{p}\left\|w_{k}\right\|_{L^{\sigma-1}\left(A_{k}, d \nu\right)} \leq\left(a_{k}^{-1} C_{k}\right)^{p}$.

\section{Applications}

We introduce first some notation.

Given $1<p<\infty$ and $0 \leq \gamma<n$ we shall define the following classes of weights $w(x)$ in $\mathbf{R}^{n}$.

$$
\begin{gathered}
\mathbf{Z}_{\mathbf{p}, \gamma}=\left\{w: \int_{\mathbf{R}^{n}} w(x)(1+|x|)^{(\gamma-n) p} d x<+\infty\right\} \\
\mathbf{D}_{\mathbf{p}, \gamma}=\left\{w: \int_{\mathbf{R}^{n}} w(x)^{1-p^{\prime}}(1+|x|)^{(\gamma-n) p^{\prime}} d x<+\infty\right\} \\
\mathbf{D}_{\mathbf{p}, \gamma}^{*}=\left\{w: \sup _{R \geq 1} R^{(\gamma-n) p^{\prime}} \int_{|x| \leq R} w^{1-p^{\prime}}(x) d x<+\infty\right\},
\end{gathered}
$$

when $\gamma=0$, we shall write simply $\mathbf{Z}_{\mathbf{p}}, \mathrm{D}_{\mathrm{p}}$ and $\mathrm{D}_{\mathbf{p}}^{*}$.

\section{Remark.}

It is easy to check that if $p<q$ then $D_{p} \subset D_{p}^{*} \subset D_{q}^{*}, D_{p} \not \subset D_{q}$ and $D_{p}^{*} \not \subset D_{q}$, in fact the weight $v(x)=\left(1+|x|^{n}\right)^{-1}$ belongs to $D_{r}^{*}$, $1<r<\infty$ but $v \notin D_{q}$ for any $q, 1<q<\infty$. Finally the weight $\omega_{p}(x)=$ $|x|^{n(p-1)}\left(1+|x|^{n}\right)^{-p}(1+|\log | x||)^{2(p-1)}$ belongs to $D_{p}$ but $\omega_{p} \notin D_{p_{1}}^{*}$ if $p_{1}<p$. 
Analogoulsy, given $1<p<\infty, 0 \leq \gamma<n$ and a measure $\mu$ on the upper half plane $\mathbf{R}_{+}^{n+1}$, we shall consider the following classes of weigths $w(x, t)$ in $\mathbf{R}_{+}^{n+1}$,

$$
\mathbf{Z}_{\mathbf{p}, \gamma}(\mathbf{d} \mu)=\left\{w: \int_{\mathbf{R}_{+}^{n+1}} w(x, t)(1+t+|x|)^{(\gamma-n) p} d \mu(\mathcal{x}, t)<+\infty\right\}
$$

$$
\mathbf{D}_{\mathbf{p}, \gamma}(\mathbf{d} \mu)=\left\{w: \int_{\mathbf{R}_{+}^{n+1}} w(x, t)^{1-p^{\prime}}(1+t+|x|)^{(\gamma-n) p^{\prime}} d \mu(x, t)<+\infty\right\}
$$

$$
\mathbf{D}_{\mathbf{p}, \gamma}^{*}(\mathbf{d} \mu)=\left\{w: \sup _{R \geq 1} R^{(\gamma-n) p^{\prime}} \int_{|x|+t \leq R} w(x, t)^{1-p^{\prime}} d \mu(x, t)<+\infty\right\},
$$

when $\gamma=0$ we shall write $\mathbf{Z}_{\mathbf{p}}(\mathbf{d} \mu), \mathbf{D}_{\mathbf{p}}(\mathbf{d} \mu)$ and $\mathbf{D}_{\mathbf{p}}^{*}(\mathbf{d} \mu)$.

\section{A. Partial sum operators.}

Consider a homogencous function of degree 0 in $\mathbf{R}^{n}, \Omega(x)=\Omega\left(x^{\prime}\right)$, which we assume to be of class $\mathcal{C}^{(1}$ outside the origin and satisfying the cancellation property

$$
\int_{\left|x^{\prime}\right|=1} \Omega\left(x^{\prime}\right) d \sigma\left(x^{\prime}\right)=0
$$

For each $\xi \in \mathbf{R}^{n}$, we define the kernel $k_{\xi}(y)=e^{2 \pi i \xi \cdot y} \Omega\left(y^{\prime}\right)|y|^{-n}$, and the corresponding operator

$$
\begin{aligned}
T_{\xi} f(x) & =p . v \cdot \int k_{\xi}(x-y) f(y) d y= \\
& =e^{2 \pi i \xi \cdot x} p \cdot v \cdot \int_{\mathrm{R}^{n}} \Omega\left((x-y)^{\prime}\right)|x-y|^{-n} e^{-2 \pi i \xi \cdot y} f(y) d y
\end{aligned}
$$

then, we define the operator

$$
T^{*} f(x)=\sup _{\xi}\left|T_{\xi} f(x)\right|
$$

and consider the inequality

$$
\int_{\mathbf{R}^{n}}\left|T^{*} f(x)\right|^{p} u(x) d x \leq C \int_{\mathbf{R}^{n}}|f(x)|^{p} v(x) d x .
$$

We have the following 
(2.8) Theorem. Let $1<p<\infty$.

(i) If $u \in Z_{p}$ then (2.7) holds for some $v$, such that $v^{\alpha} \in Z_{p}$, for $\alpha<1$.

(ii) If $v \in D_{p} \cap D_{p_{1}}^{*}$, for some $p_{1}<p$, then (2.7) holds for some $u$, such that $u^{\alpha} \in D_{p}$, for $\alpha<1$.

When $n=1$ and $\Omega(y)=\frac{1}{\pi}$ sign $(y)$ then $T_{0}=H$ (Hilbert transform) and the partial sum operarors of the Fourier Series can be expressed in terms of $\left\{T_{r}\right\}_{r \in \mathbf{R}}$ : For each interval $I=[a, b]$

$$
S_{I} f(x)=\int_{I} \hat{f}(\xi) e^{2 \pi i x \xi} d \xi=\frac{1}{2 i}\left(T_{b} f(x)-T_{a} f(x)\right) .
$$

In this case $T^{*}$ is Carleson's maximal operator, see $[\mathbf{C}],[\mathbf{H}]$,

$$
S^{*} f(x)=\sup _{I}\left|S_{I} f(x)\right| \leq C T^{*} f(x)
$$

and we have

(2.9) Theorem. Let $1<p<\infty$.

(i) $u \in Z_{p}$ if and only if (2.7) holds for some $v$,

(ii) If $v \in D_{p} \cap D_{p_{1}}^{*}$, for some $p_{1}<p$, then (2.7) holds for some $u$. Conversely if (2.7) holds for some $u$ then $v \in D_{p}$.

(iii) both (i) and (ii) are true if we replace $T^{*}$ by $S^{*}$.

\section{B. Littlewood-Paley operators.}

Let $\varphi \in S\left(\mathbf{R}^{n}\right)$ be such that $\operatorname{supp}(\hat{\varphi}) \subset\left\{\xi \in \mathbf{R}^{n}: \frac{1}{2} \leq|\xi| \leq 2\right\}$, $\hat{\varphi}(\xi)=1$ in a neighbourhood of $|\xi|=1$, and

$$
\sum_{k \in \mathbf{Z}} \hat{\varphi}\left(2^{k} \xi\right)=1 \text { for all } \xi \neq 0 .
$$

We define the following operator

$$
\mathcal{G} f(x)=\left(\sum_{k \in \mathbf{Z}}\left|\varphi_{k} * f(x)\right|^{2}\right)^{1 / 2}
$$

where $\varphi_{k}(x)=2^{k n} \varphi\left(2^{k} x\right)$, and we consider the inequality

$$
\int_{\mathbf{R}^{n}}|\mathcal{G} f(x)|^{p} u(x) d x \leq C \int_{\mathbf{R}^{n}}|f(x)|^{p} v(x) d x
$$

then we have 
(2.11) Theorem. Let $1<p<\infty$

(i) If $u \in Z_{p}$, then (2.10) holds for some $v$, such that $v^{\alpha} \in Z_{p}$ for $\alpha<1$.

(ii) If $v \in D_{p}$, then (2.10) holds for some $u$, such that $u^{\alpha} \in D_{p}$ for $\alpha<1$.

\section{Operators on the upper half plane.}

We shail consider the upper half plane $\mathbf{R}_{+}^{n+1}=\mathbf{R}^{n} \times[0, \infty)$.

Given a measure $d \nu$ on $\mathbf{R}_{+}^{n+1}$, we shall say, as usual, that $d \nu$ is a Carleson measure if there exists a constant $C$ such that for any cube $Q$ in $\mathbf{R}^{n}, \nu(\hat{Q}) \leq C|Q|$ where $\hat{Q}=\{(x, t): x \in Q, 0 \leq t \leq$ side length of $Q\}$ and $|Q|$ stands for the Lebesgue measure of $Q$.

Given a measure $d \mu$ on $\mathbf{R}_{+}^{n+1}$, we shall consider the following operators:

(2.12) Generalized fractional integrals. Let $0<\gamma<n$ and

$$
K_{\gamma}(x, t, u)=c_{n}(|x|+t+u)^{\gamma-n}, \quad x \in \mathbf{R}^{n}, \quad t, u \in[0, \infty) .
$$

Then we define, for compactly supported $f$ on $\mathbf{R}_{+}^{n+1}$

$$
T_{\mu, \gamma} f(x, t)=\int_{\mathbf{R}_{+}^{n+1}} K_{\gamma}(x-y, t, u) f(y, u) d \mu(y, u), \quad x \in \mathbf{R}^{n}, \quad y \geq 0
$$

If $f$ is a compactly supported function on $\mathbf{R}^{n}$ and $K_{\gamma}(x, t)=c_{n}(|x|+$ $t^{\gamma-n}, x \in \mathbf{R}^{n}, t \in[0, \infty)$, we define

$$
T_{\gamma} f(x, t)=\int_{\mathbf{R}^{n}} K_{\gamma}(x-y, t) f(y) d y, \quad(x, t) \in \mathbf{R}^{n+1}
$$

(2.13) Generalized Poisson integrals and Balayages.

Let $K$ be a function $K: \mathbf{R}^{n} \times \mathbf{R}^{n} \times[0, \infty) \times[0, \infty) \rightarrow \mathbf{R}^{+}$that satisfies

$$
K(x, y, t, u) \leq \frac{c_{n}}{(|x-y|+t+u)^{n}}, \quad x, y \in \mathbf{R}^{n}, t, u \in[0, \infty),
$$

we define the operator

$$
T_{\mu, 0} f(x, t)=p . v . \int_{\mathbf{R}_{+}^{n+1}} K(x, y, t, u) f(y, u) d \mu(y, u), \quad(x, t) \in \mathbf{R}_{+}^{n+1} .
$$


(2.14) Maximal operators. Let $0 \leq \gamma<n$, we define, for functions $f$ defined on $\mathbf{R}_{+}^{n+1}$, the following maximal operator $\mathcal{M}_{\mu, \gamma} f(x, t)=$ $\sup \left\{|Q|^{\frac{y}{n}-1} \int_{\hat{Q}}|f(y, s)| d \mu(y, s)\right\},(x, t) \in \mathbf{R}_{+}^{n+1}$, where the supremum is taken over the cubes $Q$ in $\mathbf{R}^{n}$ centered at $x$, with sides parallel to the axes and having side length at least $t$.

For functions $f$ defined on $\mathbf{R}^{n}$ we define

$$
\mathcal{M}_{\gamma} f(x, t)=\sup \left\{|Q|^{\frac{2}{n}-1} \int_{Q}|f(y)| d y\right\} . \quad(x, t) \in \mathbf{R}^{n+1}
$$

where the supremum is taken over the cubes $Q$ in $\mathbf{R}^{n}$ centered at $x$, with sides paratell to the axes and having side length at least $t$.

We consider the inequality

$$
\int_{\mathrm{R}_{+}^{n+1}}|T f(x, t)|^{p} u(x, t) d \nu(x, t) \leq C \int_{\mathbf{R}_{+}^{n+1}}|f(x, t)|^{p} v(x, t) d \mu(x, t)
$$

(2.16) Theorem. Let $1<p<\infty, 0 \leq \gamma<n$. Let $d \nu, d \mu$ be two

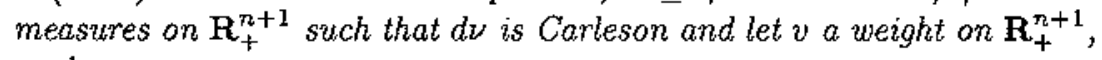
we have

(i) if $v \in D_{p, \gamma}(d \mu)$ then (2.15) holds for the operator $T_{\mu, \gamma}$ for some $u$ such that $u^{\alpha} \in D_{p, \gamma}(d \nu)$ for $\alpha<1$,

(ii) $v \in D_{p_{+} \gamma}^{*}(d \mu)$ if and only if (2.15) holds for the operator $\mathcal{M}_{\mu, \gamma}$ with $u$ such that $u^{\alpha} \in D_{p, \gamma}(d \nu)$.

(2.17) Theorem. Let $\mathrm{I}<p<\infty, 0 \leq \gamma<n$. Let $d \mu$ and $d \nu$ be two measures on $\mathbf{R}_{+}^{n+1}, u(x, t)$ be a weight on $\mathbf{R}_{+}^{n+1}$. Assume that $d \mu$ is a Carleson measure, then we have

(i) If $u \in Z_{p, \gamma}(d \nu)$ then (2.15) holds for $T_{\mu, \gamma}$ with some $v$ such that $v^{\alpha} \in Z_{p, \gamma}(d \mu), \alpha<1$.

(ii) $u \in Z_{p, \gamma}(d \nu)$ if and only if (2.15) holds for $\mathcal{M}_{\mu, \gamma}$ with some $v$ such that $v^{\alpha} \in Z_{p, \gamma}(d \mu), \alpha<1$.

We would like to emphasize some particular cases of the above results. (2.18) Given a function $g: \mathbf{R}^{n} \rightarrow \mathbf{R}$ we consider its Poisson integral

$$
P g(x, t)=c_{n} \int_{\mathbf{R}^{n}} \frac{t}{\left(|x-y|^{2}+t^{2}\right)^{\frac{n+2}{2}}} g(y) d y, \quad(x, t) \in \mathbf{R}_{+}^{n+1},
$$


on the other hand given a measure $d \mu$ on $\mathbf{R}_{+}^{n+1}$ the balayage of $d \mu$ is defined by

$$
P^{*}(d \mu)(x)=\int_{\mathbf{R}_{+}^{n+1}} \frac{c_{n} t}{\left(|x-y|^{2}+t^{2}\right)^{\frac{n+1}{2}}} d \mu(y, t), x \in \mathbf{R}^{n},
$$

$\operatorname{see}[\mathbf{A}, \mathbf{B}]$.

We have the following results

(2.19) Corollary. Let $1<p<\infty$

(i) If $v$ is a weight in $\mathbf{R}^{n}$ such that $v \in D_{p}$, then for any Carleson measure d $\nu$ there exists a weight $u$ in $\mathbf{R}_{+}^{n+1}$ such that

$$
\int_{\mathbf{R}_{+}^{n+1}}|P g(x, t)|^{p} u(x, t) d \nu(x, t) \leq C \int_{\mathbf{R}^{n}}|g(x)|^{p} v(x) d x
$$

and $u^{\alpha} \in D_{p}(d \nu)$ for $\alpha<1$.

(ii) If $u$ is a weight in $\mathbf{R}^{n}$ such that $u \in Z_{p}$ then for any Carleson measure $d \mu$ there exist a weight $v$ such that

$$
\int_{\mathbf{R}^{n}} P^{*}(f d \mu)^{p}(x) u(x) d x \leq C \int_{\mathbf{R}_{+}^{n+1}}|f(x, t)|^{p} v(x, t) d \mu(x, t),
$$

and $v^{\alpha} \in Z_{p}(d \mu)$, for $\alpha<1$.

(iii) Given a measure $d \nu$ in $\mathbf{R}_{+}^{n+1}$ and a weight $u \in Z_{p}(d \nu)$ then there exists a weight $v$ such that (2.20) holds and $v^{\alpha} \in Z_{p}$, for $\alpha<1$.

(iv) Given a measure $d \mu$ in $\mathbf{R}_{+}^{n+1}$ and a weight $v \in D_{p}(d \mu)$ then there exists a weight $u$ such that (2.21) holds and $u^{\alpha} \in D_{p}$, for $\alpha<1$.

Proof of Corollary (2.19):

Proof of parts (i) and (iii). Take the measure $d \mu(x, t)=d x \otimes \delta_{0}(t)$, where $\delta_{0}(t)$ is the Dirac's delta at $t=0$. Given a function $g(x)$ we consider the function $f(x, u)=g(x)$ then

$$
T_{\mu, 0} f(x, t)=P g(x, t),
$$

with

$$
K_{\mu, 0}(x, y, t, s)=\frac{c_{n} t}{\left(|x-y|^{2}+t^{2}\right)^{\frac{n+1}{2}}},
$$

now (i) follows from Theorem (2.16.i) and (iii) follows from Theorem (2.17.i). 
For the proof of (ii) and (iv), we consider the measure $d \nu(x, t)=d x \otimes$ $\delta_{0}(t)$ and then (ii) follows from Theorem (2.17.i) and (iv) follows from Theorem (2.16.i), since $P^{*}(f d \mu)(x)=T_{\mu, 0} f(x, t)$ with $K_{\mu, 0}(x, y, t, u)=$ $\frac{c_{n} u}{\left(|x-y|^{2}+u^{2}\right)^{\frac{n+1}{2}}}$.

(2.22) Remark. This last Corollary should be compared with the characterization, see $[\mathbf{S}]$, of the pairs $(v, d \nu)$ of weight and measure such that the Poisson integral maps $L^{p}\left(\mathbf{R}^{n}, v\right)$ into $L^{p}\left(\mathbf{R}_{+}^{n+1}, d \nu\right)$.

(2.23) Remark. It is clear that if we take $d \mu(x, t)=d x \otimes \delta_{0}(t)$ we get the operator

$$
\mathcal{M}_{\gamma} f(x)=\sup \left\{|Q|^{\frac{\gamma}{n}-1} \int_{Q}|f(y)| d y\right\}
$$

in particular

$$
\mathcal{M}_{\gamma} f(x, 0)=M_{\gamma} f(x)=\sup _{x \in Q} \frac{1}{|Q|^{1-\gamma / n}} \int_{Q}|f(y)| d y
$$

On the other hand

$$
\mathcal{M}_{\mu, \gamma} f(x, 0)=\sup _{x \in Q}\left\{|Q|^{\frac{\gamma}{n}-1} \int_{\hat{Q}}|f(y, u)| d \mu(y, u)\right\}
$$

in particular

$$
\mathcal{M}_{\mu, 0} f(x, 0)=C f(x)=\sup _{x \in Q} \frac{1}{|Q|} \int_{\hat{Q}}|f(y, u)| d \mu(y, u) .
$$

Therefore if we call $T$ to any operator $\mathcal{M}_{\gamma}, M_{\gamma}$ or $C$, then Theorems (2.16) and (2.17) give necessary and suficient conditions on the weight $v$ (resp. $u$ ) such that

$$
\int|T f|^{p} u \leq \int|f|^{p} v
$$

is satisfied for some $u$ (resp. $v$ ).

The case $M_{\gamma}$ were studied for Rubio de Francia, see [R de F, 1]. 


\section{U.M.D. Banach spaces.}

The class of the Banach spaces $E$ such that the Hilbert transform

$$
H f(x)=p . v \cdot \int_{\mathbf{R}} \frac{f(y)}{x-y} d y
$$

is bounded from $L_{E}^{2}(\mathbf{R})$ into $L_{E}^{2}(\mathbf{R})$ were characterized by Burkholder and Bourgain, see $[\mathbf{B k}],[\mathrm{B}]$, and it is denoted by U.M.D.

If $E$ is a Banach lattice of functions with absolute value $|\cdot|$, then the following extension of the Hardy-Littlewood maximal operator can be defined

$$
\tilde{M} f(x)=\sup _{x \in Q} \frac{1}{|Q|} \int_{Q}|f(y)| d y .
$$

It is known. see [R de $\mathbf{F}, 2]$, that a Banach lattice of functions $E$ is U.M.D. if and only if $\tilde{M}$ maps $L_{E}^{p}\left(\mathbf{R}^{n}\right)$ into $L_{E}^{p}\left(\mathbf{R}^{n}\right)$ and $L_{E^{*}}^{p}\left(\mathbf{R}^{n}\right)$ into $L_{E^{-}}^{p}\left(\mathbf{R}^{n}\right)$ for some $p, 1<p<\infty$, and $E^{*}$ is the dual space of $E$.

(2.24) Theorem. Let $1<p<\infty$

(i) A weight $v$ in $\mathbf{R}$ belongs to $D_{p}$ if and only if there exists $u, u^{\alpha} \in$ $D_{p}$ for $\alpha<1$ and such that for any U.M.D. Banach space $E$, we have,

$$
\int_{\mathbf{R}}\|H f(x)\|_{E}^{p} u(x) d x \leq C \int_{\mathbf{R}}\|f(x)\|_{E}^{p} v(x) d x .
$$

(ii) A weight $u$ in $\mathrm{R}$ belongs to $Z_{p}$ if and only if there exists $v, v^{\alpha} \in Z_{p}$, for $\alpha<1$ and such that (2.25) holds for every U.M.D. Banach space $E$.

(2.26) Theorem. Let $1<p<\infty$

(i) If a weight $v$ in $\mathbf{R}^{n}$ belongs to $D_{p}$ then there exists $u, u^{\alpha} \in D_{p}$ for $\alpha<1$, such that

$$
\int_{\mathbf{R}^{n}}\|\tilde{M} f(x)\|_{E}^{p} u(x) d x \leq C \int_{\mathbf{R}^{n}}\|f(x)\|_{E}^{p} v(x) d x
$$

holds for any U.M.D. Banach lattice of functions $E$. In order to satisfy (2.27) it is necessary that $v \in D_{p}^{*}$.

(ii) A weight $u$ in $\mathbf{R}^{n}$ belongs to $Z_{p}$, if and only if there exists $v$, $v^{\alpha} \in Z_{p}$ for $\alpha<1$, such that (2.27) holds for any U.M.D. Banach lattice of functions $E$. 


\section{Proofs}

The main ingredient will be to show that the corresponding operator satisfies inequality (1.2).

\section{A. Partial sums operators.}

We consider the $\ell^{\infty}$-valued operator

$$
T f(x)=\left\{p . v . \int_{\mathbf{R}^{n}} \frac{\left.\Omega(x-y)^{\prime}\right)}{|x-y|^{n}} e^{2 \pi i \xi \cdot y} f(y) d y\right\}_{\xi \in \mathrm{R}^{n}},
$$

observe that $T^{*} f(x)=\|T f(x)\|_{2 \infty}$. Therefore in order to prove Theorem $(2.8)$ it is enough to find conditions en $u$ and $v$ in order to satisfy

$$
\int_{\mathbf{R}^{n}}\|T f(x)\|_{\ell \infty}^{p} u(x) d x \leq C \int_{\mathbf{R}^{n}}|f(x)|^{p} v(x) d x .
$$

It is known by Carleson's Theorem, see [C], that $T$ maps $L^{s}\left(\mathbf{R}^{n}\right)$ into $L_{\ell \infty}^{s}\left(\mathbf{R}^{n}\right), 1<s<\infty$. On the other hand $T$ is given by the $\ell^{\infty}$-kernel

$$
\begin{gathered}
K(x, y)=\left\{\frac{\Omega\left((x-y)^{\prime}\right)}{|x-y|^{n}} e^{-2 \pi i \xi \cdot y}\right\}_{\xi}, \quad \text { i.e. } \\
T f(x)=p . v . \int K(x, y) f(y) d y,
\end{gathered}
$$

with these observations it can be proved, see [R de F, R, T], that the operator defined by

$$
\left(f_{j}\right)_{j} \rightarrow\left(T f_{j}\right)_{j}
$$

maps $L_{\ell^{p}}^{s}\left(\mathbf{R}^{n}\right)$ into $L_{\ell^{p}\left(\ell^{\infty}\right)}^{s}\left(\mathbf{R}^{n}\right), 1<p, s<\infty$.

We can consider also the transpose operator $\tilde{T}$, acting on $\ell^{1}$-valued functions $f(x)=\left(f_{\xi}(x)\right)_{\xi}$, defined by

$$
\tilde{T} f(x)=\tilde{T}\left(\left(f_{\xi}\right)_{\xi}\right)(x)=\sum_{\xi} p . v \cdot \int_{\mathbb{R}^{n}} \frac{\Omega\left((x-y)^{\prime}\right)}{|x-y|^{n}} e^{2 \pi i \xi \cdot x} f_{\xi}(y) d y .
$$

The operator $\tilde{T}$ is given by the $\ell^{\infty}=\mathcal{L}\left(\ell^{1}, \mathrm{C}\right)$-valued kernel

$$
\tilde{K}(x, y)=\left\{\frac{\Omega\left((x-y)^{\prime}\right)}{|x-y|^{n}} e^{2 \pi i \xi \cdot x}\right\} \xi \in \mathbf{R},
$$

and again the operator defined by

$$
\left(f_{j}\right)_{j} \rightarrow\left(\tilde{T} f_{j}\right)_{j}
$$


is bounded from $L_{\ell^{p}\left(\ell^{1}\right)}^{s}\left(\mathbf{R}^{n}\right)$ into $L_{\ell^{p}}^{s}\left(\mathbf{R}^{n}\right), 1<p, s<\infty$, and from $L_{\ell^{p}\left(\ell^{1}\right)}^{1}\left(\mathbf{R}^{n}\right)$ into weak- $L_{\ell^{p}}^{1}\left(\mathbf{R}^{n}\right), 1<p<\infty$, see $[\mathbf{R}$ de $\mathbf{F}, \mathbf{R}, \mathbf{T}]$.

On the other hand it is clear that $u \in Z_{p}$ if and only if $u^{1-p^{\prime}} \in$ $D_{p^{\prime}}$, hence a simply duality argument says that (i) in Theorem (2.8) is equivalent to the following statement:

(i)' If $v \in D_{p}, 1<p<\infty$, then

$$
\int_{\mathbf{R}^{n}}|\tilde{T} f(x)|^{p} u(x) d x \leq C \int_{\mathbf{R}^{n}}\|f(x)\|_{\ell^{k}}^{p} v(x) d x
$$

for some $u$, such that $u^{\alpha} \in D_{p}$, for $\alpha<1$.

Now in order to apply Theorem (1.1) to the operators $\tilde{T}$ and $\tilde{T}$ we shall need the following

(3.3) Proposition. Let $0<s<1<p$

(i) Assume that $v \in D_{p}$, then we have

$$
\left\|\left(\sum_{j}\left|\tilde{T} f_{j}\right|^{p}\right)^{1 / p}\right\|_{L^{s}\left(S_{k}\right)} \leq C_{s, p} 2^{k n / s}\left(\sum_{j}\left\|f_{j}\right\|_{L_{\ell^{1}}^{p}(v)}^{p}\right)^{1 / p}
$$

(ii) Assume that $v \in D_{p} \cap D_{p_{1}}^{*}$, for some $p_{1}<p$, then we have

$$
\left\|\left(\sum_{j}\left\|T f_{j}\right\|_{\ell^{\infty}}^{p}\right)^{1 / p}\right\|_{L^{s}\left(S_{k}\right)} \leq C_{s, p} 2^{k n / s}\left(\sum_{j}\left\|f_{j}\right\|_{L^{p}(v)}^{p}\right)^{1 / p}
$$

$k=0,1,2, \ldots$, where $S_{0}$ is the unit ball and each $S_{k}, k \geq 1$, is the spherical shell:

$$
S_{k}=\left\{x: 2^{k-1} \leq|x|<2^{k}\right\}
$$

Proof: Given $k \geq 0$, we decompose each function $f$ as $f=f^{\prime}+f^{\prime \prime}$, where $f^{\prime}=f \mathcal{X}_{B_{k}}, f^{\prime \prime}=f-f^{\prime}$ and $B_{k}=\left\{x:|x|<2^{k+1}\right\}$.

It is clear that $\|\tilde{K}(x, y)\|_{\ell_{\infty}} \leq C|x|^{-\pi}$ when $|x|>2|y|$, and therefore for all $x \in S_{k}$.

$$
\begin{aligned}
\left|\tilde{T} f^{\prime \prime}(x)\right| & \leq \int_{|y| \geq 2^{k+1}>2|x|}|\bar{K}(x, y) f(y)| d y \leq \int_{|y|>2^{k+1}}\|f(y)\|_{\ell^{1}}|y|^{-n} d y \\
& \leq C\left(\int_{\mathrm{R}^{n t}}\|f(y)\|_{\ell^{1}}^{p} v(y) d y\right)^{1 / p}\left(\int_{\mathrm{R}^{n}} \frac{v(y)^{1-p^{\prime}}}{(1+|y|)^{n p^{\prime}}} d y\right)^{1 / p^{\prime}} \\
& \leq C\|f\|_{L_{\ell^{1}}^{p}(v)},
\end{aligned}
$$


where in the last inequality we have used that $v \in D_{p}$, in particular we have

$$
\sup _{x \in S_{k}}\left(\sum_{j}\left|\tilde{T} f_{j}^{\prime \prime}(x)\right|^{p}\right)^{1 / p} \leq C\left(\sum_{j}\left\|f_{j}\right\|_{L_{\ell^{1}}^{p}(v)}^{p}\right)^{1 / p},
$$

hence

$$
\left\|\left(\sum_{j}\left|\tilde{T} f_{j}^{\prime \prime}\right|^{p}\right)^{1 / p}\right\|_{L^{s}\left(S_{k}\right)} \leq C 2^{k n / s}\left(\sum_{j}\left\|f_{j}\right\|_{L_{\ell^{1}}^{p}(v)}^{p}\right)^{1 / p} .
$$

On the other hand, as we said before, $\tilde{T}$ maps $L_{\ell^{p}\left(\ell^{1}\right)}^{1}$ into weak- $L_{\ell^{p}}^{1}$ therefore we use Cotlar's inequality (see [GC, R de F, V. 2.8]) and we get

$$
\begin{aligned}
& \left\|\left(\sum_{j}\left|\tilde{T} f_{j}^{\prime}\right|^{p}\right)^{1 / p}\right\|_{L^{s}\left(S_{k}\right)} \leq\left|S_{k}\right|^{1 / s-1}\left\|\left(\sum_{j}\left|\tilde{T} f_{j}^{\prime}\right|^{p}\right)^{1 / p}\right\|_{\text {weak }-L^{1}\left(S_{k}\right)} \\
& \leq C_{p}\left|S_{k}\right|^{1 / s-1}\left\|\left(\sum_{j}\left\|f_{j}^{\prime}\right\|_{\ell^{1}}^{p}\right)^{1 / p}\right\|_{L^{1}} \\
& \leq C_{p}\left|S_{k}\right|^{1 / s-1}\left(\int_{\mathbf{R}^{n}}\left(\sum_{j}\left\|f_{j}(x)\right\|_{\ell^{1}}^{p}\right) v(x) d x\right)^{1 / p}\left(\int_{|x|<2^{k+1}} v(x)^{1-p^{\prime}} d x\right)^{1 / p^{\prime}} \\
& \leq C_{p} 2^{k n / s}\left(\sum_{j}\left\|f_{j}\right\|_{L_{\ell^{1}}^{p}}^{p}\right)^{1 / p}
\end{aligned}
$$

where in the last inequality we have used that $v \in D_{p}$. This finishes the proof of (i).

In order to prove (ii) we decompose again each function as before $f=f^{\prime}+f^{\prime \prime}$.

Since $\|K(x, y)\|_{\ell^{\infty}} \leq C|x|^{-n}$ and $v \in D_{p}$, we have analogously as for $\tilde{T}$, that

$$
\left\|\left(\sum_{j}\left\|T f_{j}^{\prime \prime}\right\|_{\ell \infty}^{p}\right)^{1 / p}\right\|_{L^{*}\left(S_{k}\right)} \leq C 2^{k n / s}\left(\sum_{j}\left\|f_{j}\right\|_{L^{p}(v)}^{p}\right)^{1 / p}
$$


On the other hand $T$ maps $L_{\ell^{p}}^{r}\left(\mathbf{R}^{n}\right)$ into $L_{\ell^{p}\left(\ell^{\infty}\right)}^{r}\left(\mathbf{R}^{n}\right), 1<r, p<\infty$, therefore by Hölder's inequality we have

$$
\begin{aligned}
& \left\|\left(\sum_{j}\left\|T f_{j}^{\prime}\right\|_{\ell^{\infty}}^{p}\right)^{1 / p}\right\|_{L^{s}\left(S_{k}\right)} \leq\left|S_{k}\right|^{\frac{1}{s}-\frac{1}{r}}\left\|\left(\sum_{j}\left\|T f_{j}^{\prime}\right\|_{\ell^{\infty}}^{p}\right)^{1 / p}\right\|_{L^{r}\left(S_{k}\right)} \\
& \leq C\left|S_{k}\right|^{\frac{1}{g}-\frac{1}{r}}\left\|\left(\sum_{j}\left|f_{j}^{\prime}\right|^{p}\right)^{1 / p}\right\|_{L^{r}} \\
& \leq C\left|S_{k}\right|^{1 / s}\left(\int_{\mathrm{R}^{n}}\left(\sum_{j}\left|f_{j}\right|^{p}\right) v(x) d x\right)^{1 / p}\left(\int_{|x|<2^{k+1}} v(x)^{-\left(\frac{\tau}{p}\right)\left(\frac{p}{r}\right)^{\prime}}\right)^{\frac{1}{\left(\frac{p}{)^{2}\right)_{r}}\right.}}
\end{aligned}
$$

Now we choose $\tau$ such that $\frac{p}{r}=p_{1}$ and by using the hypothesis $v \in$ $D_{p_{1}}^{*}$, we have

$$
\left\|\left(\sum_{j}\left\|T f_{j}^{\prime}\right\|_{\ell^{\infty}}^{p}\right)^{1 / p}\right\|_{L^{s}\left(S_{k}\right)} \leq C 2^{k n / s}\left(\sum_{j}\left\|f_{j}\right\|_{L^{p}(v)}^{p}\right)^{1 / p} .
$$

this finishes the proof of (ii).

Proof of Theorem (2.8):

As we said above in order to prove (i) it is enough to prove (3.2). We observe that by the last Proposition the operator $\tilde{T}$ satisfies (1.2) with $F=\mathbf{R}, A_{k}=S_{k}, C_{k}=C 2^{k n / s}$ and $G=L_{\ell^{1}}^{p}(v)$, then by Theorem (1.1) there exists a weight $u$ satisfying (3.2).

Moreover $u$ can be found such that

$$
\left(\int_{S_{k}} u(x)^{1-\sigma} d x\right)^{1 / \sigma-1} \leq\left(a_{k}^{-1} 2^{k n / s}\right)^{p}
$$

with $\sigma=\left(\frac{p}{s}\right)^{\prime}$ and $a_{k}$ such that $\sum a_{k}^{p}<\infty$.

Therefore if we take $q, 1<q<\infty$, such that $q-1<p^{\prime}-1$, we have

$$
\begin{aligned}
& \int_{\mathbf{R}^{n}} \frac{u(x)^{-(q-1)}}{(1+|x|)^{n p^{\prime}}} d x \leq \sum_{K=0}^{\infty} 2^{-K n p^{\prime}} \int_{S_{K}} u(x)^{q-1} d x \leq \\
& \leq \sum_{K=0}^{\infty} 2^{-K n p^{\prime}}\left(a_{K}^{-1} 2^{k n / s}\right)^{p(q-1)}\left(2^{k n}\right) \frac{1}{\left(\frac{\sigma-1}{q-1}\right)},
\end{aligned}
$$


but $-p^{\prime}+\frac{p}{s}(q-1)+\frac{1}{\left(\frac{\sigma-1}{q-1}\right)^{\prime}}=\left(1-p^{\prime}\right)+(q-1)<0$, then if we chose $a_{K}=2^{K n \varepsilon}$, with $\varepsilon$ small enough, we get that $u^{\alpha} \in D_{p}$ with $\alpha=\frac{q-1}{p^{\prime}-1}$.

Analogously in order to prove (ii) we observe that by using Proposition (3.3) (ii) $T$ satisfies (1.2) with $F=\ell^{\infty}, A_{k}=S_{k}, C_{k}=C 2^{k n / s}$ and $G=L^{p}(v)$, then Theorem (1.1) can be applied as before.

Proof of Theorem (2.9):

The sufficient conditions on (i) and (ii) have been proved in Theorem (2.8). In order to obtain the necessary conditions we observe that

$$
T^{*} f(x) \geq|H f(x)|
$$

where $H$ is the Hilbert transform, then the conditions in order to have (2.7) are also necessary in order to have

$$
\int_{\mathbf{R}}|H f(x)|^{p} u(x) d x \leq C \int_{\mathbf{R}}|f(x)|^{p} v(x) d x,
$$

but it is well known that in order to have (3.4) for some $u$ (resp. some $v$ ) it is necessary that $v \in D_{p}$ (resp. $u \in Z_{p}$ ), see [GC, $\mathbf{R}$ de $\mathbf{F}$ ].

Finally to prove (iii) we observe that as $S^{*} f(x) \leq C T^{*} f(x)$ we have that the sufficient conditions for weigths in order to have (2.7) are also sufficient for

$$
\int_{\mathbf{R}}\left|S^{*} f(x)\right|^{p} u(x) d x \leq C \int_{\mathbf{R}}|f(x)|^{p} v(x) d x .
$$

On the other hand observe that an inequality of the type (3.5) implies that for any interval $I \subset \mathbf{R}$, the inequality

$$
\int_{\mathbf{R}}\left|S_{I} f(x)\right|^{p} u(x) d x \leq C \int_{\mathbf{R}}|f(x)|^{p} v(x) d x
$$

holds with $C$ independent of $I$ and hence (3.4) holds and the necessity conditions again are the some that they are for the Hilbert transform.

\section{B. Littlewood-Paley operators.}

Our idea is to prove Theorem (2.11) following the lines of the proof of Theorem (2.8).

We consider the $\ell^{2}$-valued operator

$$
T f(x)=\left\{\varphi_{k} * f(x)\right\}_{k \in Z}
$$


where $\varphi_{k}$ are the functions defined in section 2 part B. It is clear that $\mathcal{G} f(x)=\|T f(x)\|_{\ell^{2}}$ and then we are going to deal with the following inequality

$$
\int_{\mathbf{R}^{n}}\|T f(x)\|_{\ell^{2}}^{p} u(x) d x \leq C \int_{\mathbf{R}^{n}}|f(x)|^{p} v(x) d x
$$

in instead of (2.10).

It is known that $T$ is given by the $\ell^{2}$-valued kernel $K(x, y)=\left\{\varphi_{k}(x-\right.$ y) $\}_{k \in Z}$ that satisfies

$$
\|K(x, y)\|_{\ell^{2}} \leq C|x-y|^{-n} .
$$

Moreover the operator defined by $\left(f_{j}\right)_{j} \rightarrow\left(T f_{j}\right)_{j}$ is bounded from $L_{\ell^{p}}^{1}\left(\mathbf{R}^{n}\right)$ into weak- $L_{\ell^{p}\left(\ell^{2}\right)}^{1}\left(\mathbf{R}^{n}\right), 1<p<\infty$, see $[\mathbf{R}$ de $\mathbf{F}, \mathbf{R}, \mathbf{T}]$.

We can consider also the adjoint operator $\tilde{T}$, acting on $\ell^{2}$-valued functions, $f(x)=\left(f_{k}(x)\right)_{k}$, and defined by

$$
\tilde{T} f(x)=\tilde{T}\left(\left(f_{k}\right)_{k}\right)(x)=\sum_{k} \varphi_{k} * f(x)
$$

$\tilde{T}$ is defined by the $\ell^{2}=\mathcal{L}\left(\ell^{2}, \mathrm{C}\right)$-valued kernel

$$
\tilde{K}(x, y)=\left\{\varphi_{k}(y-x)\right\}_{k}
$$

and again the operator $\tilde{T}\left(\left(f_{j}\right)_{j}\right)$ is bounded from $L_{\ell^{p}\left(\ell^{2}\right)}^{1}$ into weak- $L_{\ell^{p}}^{1}$, $1<p<\infty$.

Therefore (i) and (ii) of Theorem (2.11) are equivalent statements and it is enough to prove (ii).

In order to prove (ii) we need the following

(3.7) Proposition. Let $v \in D_{p}, 1<p<\infty$ and let $s<1<p$. Then we have

$$
\left\|\left(\sum_{j}\left\|T f_{j}\right\|_{\ell^{2}}^{p}\right)^{1 / p}\right\|_{L^{s}\left(S_{K}\right)} \leq C_{s, p} 2^{K n / s}\left(\sum_{j}\left\|f_{j}\right\|_{L^{p}(v)}^{p}\right)^{1 / p}
$$

$K=0,1,2, \ldots$, where $S_{K}$ are th sets defined in (3.3).

The proof of this Proposition follows the pattern of the proof of Proposition (3.3).

Once we know Proposition (3.7) the proof of Theorem (2.11) can be built as the one of Theorem (2.8). 


\section{Operators on the upper half plane.}

Our goal is to establish inequality (1.2) in this context.

We shall denote by $\Gamma(x)$ the cone of aperture one whose vertex is $x$, $x \in \mathbf{R}^{n}$, i.e.

$$
\Gamma(x)=\left\{(y, t) \in \mathbf{R}_{+}^{n+1}:|x-y|<t\right\} .
$$

(3.8) Definition. Given a positive measure $d \mu$ on $\mathbf{R}_{+}^{n+1}$, we define

$$
A_{\mu} f(x)=\int_{\Gamma(x)} f(y, t) \frac{d \mu(y, t)}{t^{n}}, x \in \mathbf{R}^{n} .
$$

(3.9) Proposition. Let $1 \leq p \leq \infty$ and $d \mu$ be a measure on $\mathbf{R}_{+}^{n+1}$. Then $A_{\mu}$ is a bounded linear operator from $L_{\ell^{p}}^{1}\left(\mathbf{R}_{+}^{n+1}, d \mu\right)$ into $L_{\ell^{p}}^{1}\left(\mathbf{R}^{n}, d x\right)$.

Proof: $A_{\mu}$ is a positive linear operator, then it is enough to prove that $A_{\mu}$ maps $L^{1}\left(\mathbf{R}_{+}^{n+1} ; d \mu\right)$ into $L^{1}\left(\mathbf{R}^{n}, d x\right)$, but

$$
\begin{aligned}
\left\|A_{\mu} f\right\|_{L^{1}(d x)} & =\int_{\mathbf{R}^{n}}\left|\int_{\Gamma(x)} f(y, t) \frac{d \mu(y, t)}{t^{n}}\right| d x \\
& \leq \int_{\mathbf{R}_{+}^{n+1}}\left(\int_{\mathbf{R}^{n}} \mathcal{X}_{\Gamma(x)}(y, t)|f(y, t)| d x\right) \frac{d \mu(y, t)}{t^{n}} \\
& \leq \int_{\mathbf{R}_{+}^{n+1}}\left(\frac{1}{t^{n}} \int_{B(y, t)} d x\right)|f(y, t)| d \mu(y, t) \\
& \leq C_{n}\|f\|_{L^{1}\left(\mathbf{R}_{+}^{n+1}, d \mu\right)}
\end{aligned}
$$

(3.10) Remark. Given a positive measure $d \mu$ on $\mathbf{R}_{+}^{n+1}$, we can define the operator

$$
A_{1} f(x)=\int_{\Gamma^{\prime}(x)}|f(y, t)| \frac{d \mu(y, t)}{t^{n}}, x \in \mathbf{R}^{n}
$$

The operator $A_{1}$ is rclated with "tent spaces", see $[\mathbf{C}, \mathbf{M}, \mathbf{S}]$ and $[\mathbf{R}$, T2]. It can be showed that $A_{1}$ maps $L^{s}\left(\mathbf{R}_{+}^{n+1}, d \mu\right)$ into $L^{s}\left(\mathbf{R}^{n}, d x\right), 1<$ $s<\infty$, if only if $d \mu$ is a Carleson measure, see [R, T2]. Therefore, since $A_{\mu}(|f|)(x)=A_{1}(f)(x)$, we have that $A_{\mu}$ maps $L_{\ell^{p}}^{s}\left(\mathbf{R}_{+}^{n+1}, d \mu\right)$ into $L_{\ell^{p}}^{\delta}\left(\mathbf{R}^{n}, d x\right), 1<s<\infty, 1 \leq p \leq \infty$ if and only if $d \mu$ is a Carleson measure. 
(3.11) Proposition. Let $d \mu$ be a measure on $\mathbf{R}_{+}^{n+1}$. The following inequalities hold:

$$
\begin{aligned}
& \quad \mathcal{M}_{\mu, \gamma} f(x, t) \leq C_{n} \mathcal{M}_{\gamma}\left(A_{\mu}|f|\right)(x, t),(x, t) \in \mathbf{R}_{+}^{n+1} \\
& \quad 0 \leq \gamma<n \\
& \left|T_{\mu, \gamma} f(x, t)\right| \leq C_{n} T_{\gamma}\left(A_{\mu}|f|\right)(x, t),(x, t) \in \mathbf{R}_{+}^{n+1} \\
& 0<\gamma<n \\
& \left|T_{\mu, 0} f(x, t)\right| \leq C_{n} P\left(A_{\mu}|f|\right)(x, t),(x, t) \in \mathbf{R}_{+}^{n+1} .
\end{aligned}
$$

Where $\mathcal{M}_{\gamma}, T_{\gamma}$ and $P$ are the operators defined on (2.14), (2.12) and (2.18). By $C_{n}$ we denote a constant no necessarily the same at each ocurrence.

Proof: Let $B=B\left(x_{0}, r\right), x_{0} \in \mathbf{R}^{n}, r>0$, be a ball in $\mathbf{R}^{n}$. If $(x, t) \in \hat{B}$ then $\left|x-x_{0}\right|+t<r$, in particular we have

$$
\begin{aligned}
& |B|^{\frac{\gamma}{n}-1} \int_{\dot{B}}|f(y, u)| d \mu(y, u)=c_{n}|B|^{\frac{\gamma}{n}-1} \int_{\hat{B}}|f(y, u)|\left(\frac{1}{u^{n}} \int_{B(y, u)} d z\right) d \mu(y, u) \\
& =c_{n}|B|^{\frac{\gamma}{n}}-1 \int_{R_{+}^{n+1}} \int_{\mathbf{R}} \mathcal{X}_{\hat{B}}(y, u) \mathcal{X}_{B(y, u)}(z)|f(y, u)| \frac{d z d \mu(y, u)}{u^{n}} \\
& \leq c_{n}|B|^{\frac{\gamma}{n}-1} \int_{B} A_{\mu}(|f|)(z) d z \leq c_{n} \mathcal{M}_{\gamma}\left(A_{\mu} f\right)(x, t) .
\end{aligned}
$$

In order to prove (3.13), we observe that

$$
\begin{aligned}
\left|T_{\mu, \gamma} f(x, t)\right| & =\left|c_{n} \int_{\mathbf{R}_{+}^{n+1}} \frac{f(y, u)}{(|x-y|+t+u)^{n-\gamma}}\left(\frac{1}{u^{n}} \int_{B(y, u)} d z\right) d \mu(y, u)\right| \\
& \leq c_{n} \int_{\mathbf{R}_{+}^{n+1}} \int_{\mathbf{R}^{n}} \frac{|f(y, u)|}{(|x-y|+t+u)^{n-\gamma}} \mathcal{X}_{B(y, u)}(z) \frac{d z d \mu(y, u)}{u^{n}},
\end{aligned}
$$

but if $z \in B(y, u)$, then $|x-z|+t \leq|x-y|+u+t$ and we have

$$
\begin{aligned}
\left|T_{\mu, \gamma} f(x, t)\right| & \leq c_{n} \int_{\mathbf{R}_{+}^{n+1}} \int_{\mathbf{R}^{n}} \frac{\mid f(y, u)}{(|x-z|+t)^{n-\gamma}} \mathcal{X}_{B(y, u)}(z) \frac{d z d \mu(y, u)}{u^{n}} \\
& =c_{n} \int_{\mathbf{R}^{n}} \frac{A_{\mu}(|f|)(z)}{(\mid x-z]+t)^{n-\gamma}} d z=c_{n} T_{\gamma}\left(A_{\mu}|f|\right)(x, t) .
\end{aligned}
$$

The proof of (3.14) es analogous.

The following Theorem can be found in $[\mathbf{R}, \mathbf{T 1}]$. 
(3.15) Theorem. Let $1<p<\infty$, and $d \nu$ be a Carleson measure on $\mathbf{R}_{+}^{n+1}$, then the operators $\mathcal{M}_{\gamma}$, with $0 \leq \gamma<n, T_{\gamma}$, with $0<\gamma<n$ and $P$ are bounded from $L_{\ell^{p}}^{1}\left(\mathbf{R}^{n}, d x\right)$ into weak- $L_{\ell^{p}}^{\frac{n}{n-\gamma}}\left(\mathbf{R}_{+}^{n+1}, d \nu\right)$ and from $L_{\ell^{p}}^{q}\left(\mathbf{R}^{n}, d x\right)$ into $L_{\ell^{p}}^{r}\left(\mathbf{R}_{+}^{n+1}, d \nu\right), \frac{n}{n-\gamma}<s<\infty, \frac{1}{q}=\frac{\alpha}{n}+\frac{1}{r}$.

Taking into account this theorem and Proposition (3.11) we shall be able to prove the following

(3.16) Theorem. Let $1<p<\infty, 0 \leq \gamma<n$ and $d \nu$ be a Carleson measure in $\mathbf{R}_{+}^{n+1}$.

Given a measure d $\mu$ in $\mathbf{R}_{+}^{n+1}$ then the operators $\mathcal{M}_{\mu, \gamma}$ and $T_{\mu, \gamma}$ are bounded from $L_{\ell^{p}}^{1}\left(\mathbf{R}_{+}^{n+1}, d \mu\right)$ into weak $L_{\ell^{p}}^{\frac{n}{n-\gamma}}\left(\mathbf{R}_{+}^{n+1}, d \nu\right)$.

Moreover if $d \mu$ is a Carleson measure, then the operators $\mathcal{M}_{\mu, \gamma}$ and $T_{\mu, \gamma}$ are bounded from $L_{\ell p}^{q}\left(\mathbf{R}_{+}^{n+1}, d \mu\right)$ into $L_{\ell p}^{T}\left(\mathbf{R}_{+}^{n+1}, d \nu\right), \frac{1}{g}=\frac{\alpha}{n}+\frac{1}{r}$.

Proof: The first part of the theorem is a direct consequence of (3.9), (3.11) and (3.15).

The second part is a consequence of $(3.10),(3.11)$ and (3.15).

(3.17) Remark. The proof of Theorm (3.15) is based in the theory of vector-valued Calderon-Zygmund Kernels. That proof is not avalaible for the case of Theorem (3.16).

Now we can prove inequality (1.2) for these operators.

(3.18) Proposition. Let $0<s<1<p<\infty, 0 \leq \gamma<n$ and let $d \nu$ a Carleson measure in $\mathbf{R}_{+}^{n+1}$.

Let $S_{K}, K=0,1,2, \ldots$, be the sets in $\mathbf{R}_{+}^{n+1}$ defined by

$$
\begin{aligned}
& S_{0}=\left\{(x, t) \in \mathbf{R}_{+}^{n+1}, \quad|x|+t<1\right\} \\
& S_{K}=\left\{(x, t) \in \mathbf{R}_{+}^{n+1}, \quad 2^{K-1} \leq|x|+t<2^{K}\right\}, K=1,2, \ldots
\end{aligned}
$$

(i) If $v \in D_{p, \gamma}(d \mu)$ and $G=L^{p}\left(\mathbf{R}_{+}^{n+1}, v d \mu\right)$ then

$$
\left\|\left(\sum_{j}\left|T_{\mu, \gamma} f_{j}\right|^{p}\right)^{1 / p}\right\|_{L^{s}\left(S_{K}, d \nu\right)} \leq C 2^{\frac{n K}{s}}\left(\sum_{j}\left\|f_{j}\right\|_{G}^{p}\right)^{1 / p} .
$$

(ii) If $v \in D_{p, \gamma}^{*}(d \mu)$ and $G=L^{p}\left(\mathbf{R}_{+}^{n+1}, v d \mu\right)$ then

$$
\|\left(\sum_{j}\left|\mathcal{M}_{\mu \gamma \gamma} f_{j}\right|^{p}\right)^{1 / p_{\|} \|_{L^{s}\left(S_{K}, d \nu\right)} \leq C 2^{\frac{n K}{s}}}\left(\sum_{j}\left\|f_{j}\right\|_{G}^{p}\right)^{1 / p} .
$$


Proof: Given $K \geq 0$, we decompose each function $f=f^{\prime}+f^{\prime \prime}$ where $f^{\prime}=f \mathcal{X}_{B_{K}}, f^{\prime \prime}=f-f^{\prime}$ and

$$
B_{K}=\left\{(x, t):|x|+t<2^{K+1}\right\}
$$

If $|y|+u>2(|x|+t)$ then

$|y|+u \leq|y|+u+4 t \leq|y|+u+2 t+|y|+u-2|x| \leq 2(|x-y|+t+u)$.

Therefore if $(x, t) \in S_{K}$ we have

$$
\begin{aligned}
& \left|T_{\mu, \gamma} f^{\prime \prime}(x, t)\right|=\left|\int_{|y|+u \geq 2^{K+1}>2(|x|+t)} K_{\gamma}(x-y, t, u) f(y, u) d \mu(y, u)\right| \\
& \leq c_{n} \int_{|y|+u \geq 2^{K+1}} \mid f(y, u) \frac{d \mu(y, u)}{(|y|+u)^{n-\gamma}} \\
& \leq c_{n}\left(\int_{\mathrm{R}_{+}^{n+1}}|f(y, u)|^{p} v(y, u) d \mu(y, u)\right)^{1 / p} \\
& \quad\left(\int_{|y|+u \geq 2^{K+1}} \frac{v(y, u)^{1-p^{\prime}}}{(|y|+u)^{(n-\gamma) p^{\prime}}} d \mu(y, u)\right)^{1 / p^{\prime}} \leq c_{n}\|f\|_{G} .
\end{aligned}
$$

Thus $\sup _{(x, t) \in S_{K}}\left(\sum_{j}\left|T_{\mu, \gamma} f_{j}(x, t)\right|^{p}\right)^{1 / p} \leq C\left(\sum_{j}\left\|f_{j}\right\|_{G}^{p}\right)^{1 / p}$ and then,

$$
\begin{aligned}
\left\|\left(\sum_{j}\left|T_{\mu, \gamma} f_{j}\right|^{p}\right)^{1 / p}\right\|_{L^{s}\left(S_{K}, d \nu\right)} & \leq C \nu\left(S_{K}\right)^{1 / s}\left(\sum_{j}\left\|f_{j}\right\|_{G}^{p}\right)^{1 / p} \leq \\
& \leq C 2^{\frac{n K}{s}}\left(\sum_{j}\left\|f_{j}\right\|_{G}^{p}\right)^{1 / p}
\end{aligned}
$$

On the other hand, as $s<\frac{n}{n-\gamma}$, we use Cotlar's inequality (see [GC, R 
de F, V.2.8]) and Theorem (3.16) to get

$$
\begin{aligned}
& \left\|\left(\sum_{j}\left|T_{\mu, \gamma} f_{j}^{\prime}\right|^{p}\right)^{1 / p}\right\|_{L^{s}\left(S_{K}, d \nu\right)} \\
& \leq C \nu\left(S_{K}\right)^{1 / s-\frac{n-\gamma}{n}}\left\|\left(\sum_{j}\left|T_{\mu, \gamma} f_{j}^{\prime}\right|^{p}\right)^{1 / p}\right\|_{L_{-}^{n-\gamma}\left(\mathbf{R}_{n}^{n+2}, d \nu\right)} \\
& \leq C 2^{K\left[\frac{n}{s}-(n-\gamma)\right]}\left\|\left(\sum_{j}\left|f_{j}^{\prime}\right|^{p}\right)^{1 / p}\right\|_{L^{1}\left(\mathbf{R}_{+}^{n+3}, d \mu\right)} \\
& =C 2^{K \frac{n}{s}} \int_{B_{K}}\left(\sum_{j}\left|f_{j}(y, u)\right|^{p}\right)^{1 / p} \frac{d \mu(y, u)}{2^{K(n-\gamma)}} \\
& \leq C 2^{K \frac{n}{s}}\left(\int \sum_{j}\left|f_{j}(y, u)\right|^{p} v(y, u) d \mu(y, u)\right)^{1 / p} \\
& \cdot\left(\int \frac{v^{1-p^{\prime}}(y, u)}{(|y|+u+1)^{n-\gamma}} d \mu(y, u)\right)^{1 / p^{\prime}} \leq C 2^{K \frac{n}{s}}\left(\sum_{j}\left\|f_{j}\right\|_{G}^{p}\right)^{1 / p}
\end{aligned}
$$

This completes the proof of (i).

In order to prove (ii), we observe that if $(x, t) \in S_{K}$, then $(x, t) \in \hat{Q}_{K}$ where $Q_{K}$ is the cube

$$
Q_{K}=\left\{y \in \mathbf{R}^{n}: y=\left(y_{1}, \ldots, y_{n}\right),\left|y_{i}\right| \leq 2^{K}, \quad i=1, \ldots, n\right\},
$$

therefore

$$
\begin{aligned}
& \frac{1}{\left|Q_{K}\right|^{\frac{\gamma}{n}-1}} \int_{\hat{Q}_{K}}\left|f^{\prime \prime}(y, u)\right| d \mu(y, u) \leq \\
& \leq C_{n}\|f\|_{G} 2^{K(\gamma-n)}\left(\int_{B_{K}} v(y, u)^{1-p^{\prime}} d \mu(y, u)\right)^{1 / p^{\prime}} \leq \\
& \leq C_{n}\|f\|_{G} \sup _{R \geq 1}\left(R^{(\gamma-n) p^{\prime}} \int_{|x|+u \leq R} v(y, u)^{1-p^{\prime}} d \mu(y, u)\right)^{1 / p^{\prime}} \leq \\
& \leq C_{n}\|f\|_{G} .
\end{aligned}
$$

Now the rest of the proof follows as in (i).

Proof of Theorem (2.16): If $v \in D_{p, \gamma}(d \mu)$, then, by the last Proposition, inequality (1.2) is satisfied for $T_{\mu, \gamma}$ with $A_{K}=S_{K}, G=$ $L^{p}(v d \mu), F=\mathbf{R}, c_{K}=2^{K \frac{\mathrm{n}}{3}}$. Therefore by Theorem (1.1) there exists $v$ satisfying (2.15) for $T_{\mu, \gamma}$. Moreover $u$ is such that

$$
\left\|u^{-1} \mathcal{X}_{S_{K}}\right\|_{L^{\sigma-1}\left(A_{K}, d \nu\right)} \leq\left(a_{K}^{-1} 2^{\frac{K n}{s}}\right)^{p}
$$


with $\sigma=\left(\frac{p}{s}\right)^{\prime}$ and $\sum a_{K}^{p}<+\infty$, then

$$
\int_{\mathbf{R}^{n+1}} \frac{u(x)^{1-\sigma}}{(1+t+|x|)^{(n-\gamma) p^{\prime}}} d \nu(x, t) \leq \sum_{K=0}^{\infty} 2^{-K(n-\gamma) p^{\prime}}\left(a_{K}^{-1} 2^{\frac{K n}{s}}\right)^{p(\sigma-1)}
$$

but as $\left(\begin{array}{l}p \\ s\end{array}\right)^{\prime}<p^{\prime}$ we have $-(n-\gamma) p^{\prime}+\frac{n}{s} p(\sigma-1)<0$.

Therefore it is enough to choose $a_{K}=2^{-K \varepsilon}$ with $\varepsilon$ small enough and then $u^{\alpha} \in D_{p_{1} \gamma}$ with $\alpha=\frac{\sigma-1}{p^{\prime}-1}$.

This finishes the proof of (i).

The proof of the suffciency of condition $D_{p, \gamma}^{*}(d \mu)$ in $(2,16)$ is obtained in a similar way using (3.18) (ii).

For the necessity observe that for any ball $B$

$$
\hat{B} \subset\left\{(x, t): \mathcal{M}_{\mu, \gamma} f(x, t) \geq|B|^{\frac{\gamma}{n}-1} \int_{\hat{B}}|f(y, u)| d \mu(y, u)\right\},
$$

then (2.15) for $T=M_{\mu, \gamma}$ implies that

$$
\begin{aligned}
& \int_{\hat{B}} u(x, t) d \mu(x, t) \leq \\
\leq & C\left(\int_{\hat{B}}|f(y, u)| d \mu(y, u)\right)^{-p}|B|^{\left(\frac{\gamma}{2}-1\right) p} \int_{\mathrm{R}_{+}^{n+1}}|f(y, u)|^{p} v(y, u) d \mu(y, u),
\end{aligned}
$$

therefore for $f=\mathcal{X}_{\hat{B}} v^{1-p^{\prime}}$ we get the result.

Proof of Theorem (2.17): Since $T_{\mu, \gamma}$ is essentially self-adjoint, a simple duality argument shows that the pair $(u(x, t), v(x, t))$ satisfies (2.15) for the exponent $p$ if and only if the pair $\left(v(x, t)^{1-p^{\prime}}, u(x, t)^{1-p^{\prime}}\right)$ satisfies the some inequality with exponent $p^{\prime}$. Thus (i) is actually equivalent to (2.16) (i).

The necessity of (ii) is obtained as in (2.16) (ii).

For the sufficiency we consider the $\ell^{\infty}$-valued operator

$$
\tilde{T}_{\mu, \gamma} f(x, t)=\left\{\mathcal{X}_{\bar{Q}_{r}}(x, t) \frac{1}{\left|Q_{r}\right|^{1-\gamma / n}} \int_{\tilde{Q}_{r}} f(y, u) d \mu(y, u)\right\}_{r \in \mathbf{R}}
$$

where $Q_{r}$ is the cube centered at origine and with side length $r$.

It is clear that $\mathcal{M}_{\mu, \gamma} f(x, t)=\left\|T_{\mu, \gamma} f(x, t)\right\|_{\ell^{\infty}}$. Therefore a pair $(u(x, t), v(x, t))$ satisfies (2.15) for $\mathcal{M}_{\mu, \gamma}$ if and only if satisfies

$$
\int_{\mathbf{R}_{+}^{n+1}}\left\|\tilde{T}_{\mu, \gamma} f(x, t)\right\|_{\ell^{\infty}}^{p} u(x, t) d \nu(x, t) \leq C \int_{\mathbf{R}_{+}^{n+1}}|f(x, t)|^{p} v(x, t) d \mu(x, t) .
$$


On the other hand we consider the operator $\tilde{S}_{\nu, \gamma}$, acting on $\ell^{1}$-valued functions $g(x, t)=\left(g_{r}(x, t)\right)_{r}$, defined by

$$
\begin{aligned}
\tilde{S}_{\nu, \gamma} g(x, t) & =\tilde{S}_{\nu_{\gamma} \gamma}\left(\left(g_{r}\right)_{r}\right)(x, t)= \\
& =\sum_{r}\left(\frac{1}{\left|Q_{r}\right|^{1-\gamma / n}} \int_{\hat{Q}_{\tau}} g_{r}(y, u) d \mu(y, u)\right) \mathcal{X}_{\hat{Q}_{r}}(x, t) .
\end{aligned}
$$

A simple duality argument shows that $(u(x, t), v(x, t))$ satisfies (3.19) if and only if $\left(v(x, t)^{1-p^{\prime}}, u(x, t)^{1-p^{\prime}}\right)$ satisfies

$$
\int\left|\tilde{S}_{\nu, \gamma} g(x, t)\right|^{p^{\prime}} v(x, t)^{1-p^{\prime}} d \mu(x, t) \leq C \int\|g(x, t)\|_{\ell^{1}}^{p^{\prime}} u(x, t)^{1-p^{\prime}} d \nu(x, t) .
$$

It is clear, see the proof of Proposition (3.11), that

$$
\left.\tilde{S}_{\nu, \gamma} g(x, t)=\tilde{S}_{v, \gamma}\left(\left(g_{r}\right)_{r}\right)(x, t) \leq \tilde{S}_{\gamma}\left(\left(A_{\nu}\left(\mid g_{r}\right]\right)\right)_{r}\right)(x, t)
$$

where

$$
\tilde{S}_{\gamma}\left(\left(f_{r}\right)_{r}\right)(x, t)=\sum_{r}\left(\frac{1}{\left|Q_{r}\right|^{1-\gamma / n}} \int_{Q_{r}} f_{r}(y) d y\right) \mathcal{X}_{\hat{Q}_{r}}(x, t),
$$

but, since $d \nu$ is a Carleson measure, $\tilde{S}_{\gamma}$ maps $L_{\ell^{p}\left(\ell^{1}\right)}^{1}(d x)$ into weak$L_{\ell^{p}}^{\frac{n}{n-\gamma}}(d \nu), 1<p<\infty$; therefore by using (3.9) we conclude that $\tilde{S}_{\nu, \gamma}$ maps $L_{\ell^{p}\left(\ell^{1}\right)}^{1}(d \nu)$ into weak- $L_{\ell^{p}}^{\frac{n}{n-\gamma}}(d \mu)$. Moreover $\tilde{S}_{\nu, \gamma}$ has a $\mathcal{L}\left(\ell^{1}, \mathbf{C}\right) \simeq$ $\ell^{\infty}$-valued kernel

$$
K(x, y, t, u)=\left\{\frac{\mathcal{X}_{\bar{Q}_{r}}(x, t)}{\left|Q_{r}\right|^{1-\gamma / n}} \mathcal{X}_{\hat{Q}_{r}}(y, u)\right\}_{r}
$$

that satisfies

$$
\|K(x, y, t, u)\| \leq \frac{C}{(|x-y|+t+u)^{n-\gamma}} .
$$

With the last two ingredients and using the ideas in the proof of (3.18) (i), one can proof that

(3.21) If $u^{1-p^{\prime}} \in D_{p^{\prime}, \gamma}(d \nu)$ and $G=L_{\ell^{1}}^{p^{\prime}}\left(\mathbf{R}_{+}^{n+1}, u^{1-p^{\prime}} d \nu\right)$ then

$$
\left\|\left(\sum_{j}\left|\tilde{S}_{\nu, \gamma} g_{j}\right|^{p^{\prime}}\right)^{1 / p^{\prime}}\right\|_{L^{\prime}\left(S_{K}, d \nu\right)} \leq C 2^{\frac{n k}{*}}\left(\sum_{j}\left\|g_{j}\right\|_{G}^{p^{\prime}}\right)^{1 / p^{\prime}} \text {. }
$$

Therefore we can apply Theorem (1.1) and conclude that there exists a weight $v^{1-p^{\prime}}$, with $v^{\alpha\left(1-p^{\prime}\right)} \in D_{p^{\prime}, \gamma}(d \mu)$ and such that (3.20) holds.

Now the proof finishes by observing that $u^{1-p^{\prime}} \in D_{p^{\prime}, \gamma}(d \nu)$ if and only if $u \in Z_{p, \gamma}(d \nu)$. 


\section{U.M.D. Banach spaces.}

Proof of the Theorem (2.24): It is known that a Banach space $E$ is U.M.D. if and only if $E^{*}$ is U.M.D., therefore, by duality, a pair satisfies (2.25) if and only if the pair $\left(v(x)^{1-p^{\prime}}, u(x)^{1-p^{\prime}}\right)$ satisfies

$$
\int_{\mathbf{R}}\|H f(x)\|_{E^{\cdot}}^{p^{\prime}} \cdot v(x)^{1-p^{\prime}} d x \leq C \int_{\mathbf{R}}\|f(x)\|_{E^{*}}^{p^{\prime}} u(x)^{1-p^{\prime}} d x .
$$

Then (i) and (ii) are equivalent statementes in Theorem (2.24) and we shall limit ourselves to prove (i).

Given a vector $e \in E$ and a function $\varphi \in L^{p}(d x)$, we consider the $E$-valued function $f(x)=\varphi(x) e$, then (2.25) implies that

$$
\|e\|_{E} \int_{\mathbf{R}}|H \varphi(x)|^{p} u(x) d x \leq C\|e\|_{E} \int_{\mathbf{R}}|\varphi(x)|^{p} v(x) d x,
$$

therefore the necessity of the condition $D_{p}$, follows from the known necessarily condition in order to have

$$
\int_{\mathbf{R}}|H \varphi(x)|^{p} u(x) d x \leq C \int_{\mathbf{R}}|\varphi(x)|^{p} v(x) d x
$$

see $[C, J],[R$ de $F, 1]$.

For the sufficiency we need the following

(3.22) Proposition. Let $v \in D_{p}, 1<p<\infty$, and $0<s<1$.

Then for every $K=0,1,2, \ldots$ we have

$$
\left\|\left(\sum_{j}\left\|H f_{j}\right\|_{E}^{p}\right)^{1 / p}\right\|_{L^{a}\left(S_{K}\right)} \leq C 2^{k / s}\left(\sum_{j}\left\|f_{j}\right\|_{L_{E}^{p}(v)}^{p}\right)^{1 / p}
$$

where $S_{0}$ is the unit ball in $\mathbf{R}$ and each $S_{K}, K \geq 1$, is the spherical shell

$$
S_{K}=\left\{x: 2^{K-1} \leq|x|<2^{K}\right\} \text {. }
$$

Proof: Given $K \geq 0$, we decompose each function as $f=f^{\prime}+f^{\prime \prime}$, where $f^{\prime}=f \mathcal{X}_{B_{K}}, f^{\prime \prime}=f-f^{\prime}$ and $B_{K}=\left\{x:|x|<2^{K+1}\right\}$.

If $x \in S_{K}$, we have

$$
\begin{aligned}
\left\|H f^{\prime \prime}(x)\right\|_{E} & \leq \int_{|y| \geq 2^{K+1}>2|x|} \frac{1}{|x-y|}\|f(x)\|_{E} d y \\
& \leq C \int_{|y| \geq 2^{K+1}}\|f(y)\|_{E}|y|^{-1} d y \\
& \leq C\|f\|_{L_{E}^{p}(v)}
\end{aligned}
$$


thus, Minkowski's inequality gives

$$
\sup _{x \in S_{K}} \sum_{j}\left\|H f_{j}^{\prime \prime}(x)\right\|_{E} \leq C\left(\sum_{j}\left\|f_{j}\right\|_{L_{E}^{p}(v)}^{p}\right)^{1 / p}
$$

On the other hand it is well known, see [Bk], that if $E$ is U.M.D. then $H$ maps $L_{E}^{1}(\mathbf{R})$ into weak $L_{E}^{1}(\mathbf{R})$ and also that if $E$ is U.M.D then $\ell_{E}^{p}$, $1<p<\infty$, is U.M.D. Then by Cotlar's inequality, see [GC, $\mathbf{R}$ de $\mathbf{F}$, V.2.8], we have

$$
\begin{aligned}
\left\|\left(\sum_{j}\left\|H f_{f}^{\prime}\right\|_{E}^{p}\right)^{1 / p}\right\|_{L^{s}\left(S_{K}\right)} & \leq C\left|S_{K}\right|^{1 / s-1}\left\|\left(\sum_{j}\left\|H f_{j}^{\prime}\right\|_{E}^{p}\right)^{1 / p}\right\|_{L_{*}^{1}(\mathrm{R})} \\
& \leq C\left|S_{K}\right|^{1 / s-1}\left\|\left(\sum_{j}\left\|f_{j}^{\prime}\right\|_{E}^{p}\right)^{1 / p}\right\|_{1} \\
& \leq C\left|S_{K}\right|^{1 / s} \int\left(\sum_{j}\left\|f_{j}(x)\right\|_{E}^{p}\right)^{1 / p} \frac{d x}{2^{K}} \\
& \leq C\left|S_{K}\right|^{1 / s}\left\|\left(\sum_{j}\left\|f_{j}\right\|_{E}^{p}\right)^{1 / p}\right\|_{L^{p}(v)} \\
& =C 2^{K / s}\left(\sum_{j}\left\|f_{j}\right\|_{L_{E}^{p}(v)}^{p}\right)^{1 / p} .
\end{aligned}
$$

Now we continue the proof of the sufficiency in Theorem (2.4). We apply Theorem (1.1) with $A_{K}=S_{K}, F=E, G=L_{E}^{p}(v)$ and we conclude that there exists $u$ satisfying (2.25). Moreover $u$ can be found such that

$$
\left(\int_{S_{K}} u(x)^{1-\sigma} d x\right) \leq\left(a_{K}^{-1} 2^{\frac{K}{s}}\right)^{p(\sigma-1)}
$$

with $\sigma=\left(\frac{p}{s}\right)^{\prime}$ and $a_{K}$ such that $\sum a_{K}^{p}<+\infty$, then

$$
\int_{\mathrm{R}} \frac{u(x)^{1-\sigma}}{(1+|x|)^{p^{\prime}}} \leq \sum_{K=0}^{\infty} 2^{-K p^{\prime}}\left(a_{K}^{-1} 2^{\frac{K}{3}}\right)^{p) \sigma-1)},
$$

but as $\sigma=\left(\frac{p}{s}\right)^{\prime}<p$ we have $-p^{\prime}+\frac{p}{s}(\sigma-1)<0$, therefore if we choose $a_{K}=2^{-K \varepsilon}$ with $\varepsilon$ small enough we obtain that $u^{\alpha} \in D_{p}$ for $\alpha=\frac{p^{t}-1}{\sigma-1}$.

Proof of (2.26): The necessity condition follows as in Theorem (2.24) since if we take $f(x)=\varphi(x) e, \varphi \in L^{p}\left(\mathbf{R}^{n}\right) e \in E$, we have

$$
\|e\|_{E} \int_{\mathbf{R}^{n}} M \varphi(x)^{p} u(x) d x \leq C\|e\|_{E} \int_{\mathbf{R}^{n}}|\varphi(x)|^{p} v(x) d x .
$$


For the sufficiency we consider the $E\left(\ell^{\infty}\right)$-valued operator

$$
\tilde{T} f(x)=\left\{\mathcal{X}_{Q_{r}}(x) \frac{1}{\left|Q_{r}\right|} \int_{Q_{r}} f(y) d y\right\}_{r \in \mathbf{R}}
$$

where $Q_{r}$ is the cube in $\mathbf{R}^{n}$ centered at origine and with side length $r$. It is clear that for positive $E$-valued functions we have

$$
\|\tilde{M} f(x)\|_{E}=\|\tilde{T} f(x)\|_{E(\xi \infty)} .
$$

Therefore a pair $(u(x), v(x))$ satisfies (2.27) if and only if satisfies

$$
\int_{\mathbf{R}^{n}}\|\tilde{T} f(x)\|_{E(\ell \infty)}^{p} u(x) d x \leq C \int_{\mathbf{R}^{n}}\|f(x)\|_{E}^{p} v(x) d x
$$

Since $E$ is a U.M.D. Banach lattice, then $\ell^{p}(E), 1<p<\infty$, is a U.M.D. Banach lattice then the operator $\left(f_{j}\right) \rightarrow\left(\tilde{M} f_{j}\right)$ is bounded from $L_{\ell^{p}(E)}^{1}\left(\mathbf{R}^{n}\right)$ into weak $-L_{\ell p(E)}^{1}\left(\mathbf{R}^{n}\right)$, and therefore the operator $\left(f_{j}\right) \rightarrow$ $\left(\tilde{T} f_{j}\right)$ is bounded from $L_{\ell^{p}(E)}^{1}\left(\mathbf{R}^{n}\right)$ into weak- $L_{\ell^{p}\left(E\left(\ell^{\infty}\right)\right)}^{1}\left(\mathbf{R}^{n}\right)$.

Moreover $\tilde{T}$ is an operator given by a $\ell^{\infty} \subseteq \mathcal{L}\left(E, E\left(\ell^{\infty}\right)\right)$-valued kernel

$$
\tilde{K}(x, y)=\left\{\frac{\mathcal{X}_{Q_{r}}(x) \mathcal{X}_{Q_{r}}(y)}{\left|Q_{r}\right|}\right\}_{r \in \mathbf{R}}
$$

This kernel obviously satisfies

$$
\|\tilde{K}(x, y)\|_{\ell_{\infty}} \leq \frac{C}{|x-y|^{n}} .
$$

With the last two observations it is easy to follow the patterns of the proof of Proposition (3.22) in order to prove

(3.24) Proposition. Let $v \in D_{p}, 1<p<\infty$, and $0<s<01$.

Then for every $K=0,1,2, \ldots$ we have

$$
\left\|\left(\sum_{j}\left\|\tilde{T} f_{j}\right\|_{E(\ell \infty)}^{p}\right)^{1 / p}\right\|_{L^{s}\left(S_{K}\right)} \leq C 2^{\frac{K n}{s}}\left(\sum_{j}\left\|f_{j}\right\|_{L_{E}^{p}(v)}^{p}\right)^{1 / p}
$$

where $S_{0}$ is the unit ball in $\mathbf{R}^{n}$ and each $S_{K}, K \geq 1$, is the spherical shell

$$
S_{K}=\left\{x \in \mathbf{R}^{n}: 2^{K-1} \leq|x|<2^{K}\right\} .
$$


Now we aply Theorem (1.1) with $A_{K}=S_{K} \cdot F=E\left(\ell^{\infty}\right), G=L_{E}^{p}(v)$ and we conclude that there exists $u$ satisfying (3.23), hence (2.27) and such that

$$
\int_{S_{K}} u(x)^{1-\sigma} d x \leq\left(a_{K}^{-1} 2^{\frac{K n}{s}}\right)^{p(\sigma-1)}
$$

with $\sigma=\left(\frac{p}{s}\right)^{\prime}$ and $a_{K}$ such that $\sum_{K} a_{K}^{p}<+\infty$, then proceeding as usual we find $u^{\alpha} \in D_{p}$ for $\alpha=\frac{p^{\prime}-1}{\sigma-1}$.

In order to prove the sufficient condition in part (ii) we consider the operator $\tilde{S}$, acting on $E^{*}\left(\ell^{1}\right)$-valued functions, $g(x)=\left(g_{r}(x)\right)_{r}$, defined by

$$
\tilde{S} g(x)=\sum_{r}\left(\frac{1}{\left|Q_{r}\right|} \int_{Q_{r}} g_{r}(y) d y\right) \mathcal{X}_{Q_{r}}(x)
$$

A simple duality argument shows that $(u, v)$ satisfies (3.23) if and only if $\left(v^{1-p^{\prime}}, u^{1-p^{\prime}}\right)$ satisfies

$$
\int_{\mathbf{R}^{n}}\|\tilde{S} g(x)\|_{E^{*}}^{p^{\prime}} v^{1-p^{\prime}}(x) d x \leq C \int_{\mathbf{R}^{n}}\|g(x)\|_{E^{*}\left(\ell^{1}\right)}^{p^{\prime}} u^{1-p^{\prime}}(x) d x
$$

but as $\ell^{p^{\prime}}\left(E^{*}\right), 1<p<\infty$, is a U.M.D. Banach lattice we have that the operator $\left(g_{j}\right)_{j} \rightarrow\left(\tilde{S} g_{j}\right)_{j}$ is bounded from $L_{\ell p^{\prime}\left(E^{*}\left(\ell^{1}\right)\right)}^{1}\left(\mathbf{R}^{n}\right)$ into weak$L_{\ell^{p^{\prime}}\left(E^{*}\right)}^{1}\left(\mathbf{R}^{n}\right)$, Moreover $\tilde{S}$ has the $\ell^{\infty} \subseteq \mathcal{L}\left(E^{*}\left(\ell^{1}\right), E^{*}\right)$-valued kernel

$$
L(x, y)=\left\{\frac{\mathcal{X}_{Q_{r}}(x) \mathcal{X}_{Q_{r}}(y)}{\left|Q_{r}\right|}\right\}_{r \in \mathbf{R}}
$$

satisfying $\|L(x, y)\|_{\ell^{\infty}} \leq \frac{C}{|x-y|^{n}}$.

Then we can reproduce the arguments that we did above for $\tilde{T}$ and obtain that if $u^{1-p^{t}} \in D_{p^{\prime}}$ then (3.25) holds for some $v^{\left(1-p^{\prime}\right) \alpha} \in D_{p^{t}}$. Therefore by duality if $u \in Z_{p}$ then (3.23), and hence (2.27), holds for some $v^{\alpha} \in Z_{p}$. 


\section{References}

[A, B] Amar, E. and Bonami, A., Measures de Carleson d'odre $\alpha$ et solutions au bord de l'equation $\bar{\partial}$, Bull. Soc. Math. France 107 (1979), 23-48.

[B] Bourgain, J., Some remarks on Banach spaces in which Martingale difference sequences are inconditional, Ark. Mot. 22 (1983), 163-168.

[Bk] BURKHOLDER, D, A geometrical characterization of Banach spaces in which martingale difference sequences are inconditional, Ann. Probability 9 (1981), 997-1011.

[C] CaRleson, C., On convergence and growth of partial sums of Fourier Series, Acta Math. 116 (1966), 135-157.

[C, J] CARLESON, L. AND JONES, P., Weighted norm inequalities and a theorem of koosis, Mittag-Leffer Institut, Report no. 2.

[C, M, S] Colfman, R. R., Meyer, Y. and Stein, E. M., Some new function spaces and their applications to Harmonic Analysis, Journal of Funct. Anolysis 62 (1985), 304-335.

[F, S] Fefrerman, C. And Stein, E. M., Some maximal inequalities, Amer. J. Math. 1 (1971), 107-115.

[GC, R de F] Garcia-Cuerva, J. and Rubio de Francia, J. L., "Weighted norm inequalities and related topics," North-Holland, Math. Studies 116, 1985.

[G, G] Gatto, A. E. And Gutierrez, C. E., On weighted norm inequalities for the maximal function, Studia Math. 76 .

[H] HUNT, R. A., "On the convergence of Fourier Series," Proceedings Conf. Orthogonal Expansions, Sothern Illinous Univ. Press, Carbondale, 1968, pp. 235-255.

[H, M, S] Harboure, E., Macias, R. A. and Segovia, C. Boundedness of fractional operators on $L^{p}$ spaces with Different weights, Trans. Amer. Math. Soc. 285 (1984), 629-647.

[ $\mathrm{R}$ de $\mathrm{F}, 1]$ RUBIO DE FRANCIA, J. L, "Weighted norm inequalities and vector-valued inequalities," Harmonic Analysis (Ricci, F. and Weiss, G. editors), Lecture Notes in Math. 908, Springer-Verlag, 1982, pp. 86-101.

[R de F, 2] RUBIO DE FRANCIA, J. L., "Martingale and integral transforms of Banach space valued functions," Probability and Banach spaces (Bastero, J. and San Miguel, M., editors), Lectures Notes in Math., 1221, Springer-Verlag, 1985, pp. 195-222. 
[R de F, R, T] Rubio de Francia, J. L., Ruiz, F. J. and Torrea, J. L., Calderón-Zygmund theory for vector valued functions, $A d v$. in Math. 62 (1986), 7-48.

[R, T1] Ruiz, F. J. AND TORREA, J. L., Weigthed and vector-valued inequalities for Potential operators, Trans. Amer. Math. Soc. 295 (1986), 213-232.

[R, T2] Ruiz, F. I. AND TORreA, J. L., Vector-valued CalderonZygmund theory applied to tent spaces, Colloquium Math. 62 (1991), 265-277.

[S] SAWYER, E., A characterization of Two Weight Norm inequalities for Fractional and Poisson integrals, Trans. Amer. Math. Soc. 308 (1988), 533-545.

Luz M. Fernández-Cabrera:

Escuela Universitaria de Estadística Universidad Complutense de Madrid Madrid

SPAIN
José L. Torrea:

Departamento de Matemáticas

Universidad Autónoma de Madrid 28049 Madrid

SPAIN

Rebut el 4 de Setembre de 1992 